\title{
AVALIAÇÃO DE EMPRESAS: Um Estudo Bibliométrico das Publicações nas Revistas Brasileiras $^{1}$
}

\section{COMPANY VALUATION: A Bibliometric Study of Publications in Brazilian Journals}

\section{EVALUACIÓN COMPAÑía: Un Estudio Bibliométrico de las Publicaciones en Revistas Brasileñas}

Eddie Raoni Marques, Mestrando em Controladoria pela

Universidade Federal Rural de Pernambuco (UFRPE). Endereço

Profissional: Universidade Federal Rural de Pernambuco (UFRPE),

Programa de Pós-Graduação em Controladoria (PPGC), Rua D. Manoel de Medeiros s/n,

Dois Irmãos, Recife, PE - Brasil, CEP 52171900. Telefone: (081) 33205403.

URL da Homepage: http://www.ppgc.ufrpe.br/?q=pt-br E-mail: eddieraoni @ hotmail.com

Glauber Quirino Falcão, Mestrando em Controladoria pela

Universidade Federal Rural de Pernambuco (UFRPE). Endereço

Profissional: Universidade Federal Rural de Pernambuco (UFRPE),

Programa de Pós-Graduação em Controladoria (PPGC), Rua D. Manoel de Medeiros s/n,

Dois Irmãos, Recife, PE - Brasil, CEP 52171900. Telefone: (081) 33205403.

URL da Homepage: http://www.ppgc.ufrpe.br/?q=pt-br E-mail: glauber.falcao@gmail.com

\section{RESUMO}

As dinâmicas do mercado demandam cada vez mais estimativas dos valores das empresas, tal procedimento é denominado Avaliação de Empresas, também conhecido como Valuation. Esse estudo teve como objetivo descrever as características em torno das publicações que tratam da temática em evidência. Para atender a esse objetivo, foi desenvolvida uma pesquisa documental, de abordagem bibliométrica, com ênfase descritiva e de caráter quantitativo. $\mathrm{O}$ universo estudado abrangeu artigos publicados entre 2011 e 2015 e que se encontram disponíveis no sistema de busca do portal de Periódicos da CAPES. Como critérios de identificação das publicações, foram selecionados os materiais que traziam os termos "avaliação de empresas" e "valuation" no título, palavras-chave e/ou resumo. Os resultados foram analisados a partir de três grupos principais: características dos periódicos, características dos autores e características dos artigos. As contribuições apresentadas permitirão nortear futuras pesquisas em torno da temática, como também contribuir com informações pertinentes para os periódicos que publicam sobre o assunto.

Palavras-chave: avaliação de empresa; bibliometria; técnicas de avaliação.

\section{ABSTRACT}

The dynamics of the market demand more and more estimates of the values of the companies; this procedure is called Valuation of Companies, also known as Valuation. The purpose of this study was to describe the characteristics of publications that deal with this issue in evidence. To achieve this objective, a documentary research was developed, with a bibliometric

\footnotetext{
${ }^{1}$ Artigo submetido em 05/03/2017, revisado em 26/05/2017, aceito em 17/11/2017 e divulgado em 15/12/2017 pelo Editor João Carlos Hipólito Bernardes do Nascimento, após double blind review.
}

GєCont, v. 4, n. 1, Floriano-PI, Jan-Jun. 2017. 
approach, under a descriptive and quantitative emphasis. The studied universe covered articles published between 2011 and 2015 and that are available in the CAPES search system Periodicals portal. As criteria for the identification of publications: materials that carried the terms "company valuation" and "valuation" in the title, keywords and/or abstract were selected. The results were analyzed from three main groups: characteristics of the journals, characteristics of the authors and characteristics of the articles. The contributions presented will guide future research around the theme, as well as contribute relevant information to the periodicals that publish on the subject.

Keywords: company evaluation; bibliometry; evaluation techniques.

\section{RESUMEN}

La dinámica del mercado demanda cada vez más estimaciones de los valores de las empresas; este procedimiento se llama Valoración de empresas, también conocido como Valoración. El propósito de este estudio fue describir las características de las publicaciones que tratan este tema como evidencia. Para lograr este objetivo, se desarrolló una investigación documental, con un enfoque bibliométrico, bajo un énfasis descriptivo y cuantitativo. El universo estudiado cubrió los artículos publicados entre 2011 y 2015 y están disponibles en el sistema de búsqueda de CAPES - Portal de publicaciones periódicas. Como criterios para la identificación de publicaciones: materiales que incluían los términos "valoración de la empresa" y "valoración" en el título, se seleccionaron palabras clave y / o resumen. Los resultados se analizaron a partir de tres grupos principales: características de las revistas, características de los autores y características de los artículos. Las contribuciones presentadas guiarán la investigación futura en torno al tema, así como también aportará información relevante a las publicaciones periódicas que publican sobre el tema.

Palabras clave: evaluación de la empresa; bibliometría; técnicas de evaluación.

\section{INTRODUÇÃO}

ão inúmeros os motivos para se efetuar uma avaliação de empresa, mas qualquer - que seja o motivo, a busca do valor da empresa é o alvo do avaliador, que certamente não terá acesso a todas as informações disponíveis, tampouco um modelo perfeito de avaliação; sendo assim, é necessário analisar se as avaliações com as informações disponíveis no momento e com o modelo existente proveem uma aproximação desse valor (CUNHA; MARTINS; NETO, 2014).

Fatores como a interferência governamental, incertezas da economia, variação das taxas de juros e aumento da competitividade tornam, para os participantes desse ambiente empresarial, a avaliação mais difícil, demandando julgamentos que atendam às particularidades de cada empresa e de cada ambiente empresarial. (PEREIRO, 2002; ASSAF NETO, 2003).

Existem várias técnicas de avaliação de empresas, as mais utilizadas têm sido: FDC Fluxo de Caixa Descontado, EVA - Valor Econômico Agregado, MVA - Valor de Mercado Adicionado e Múltiplos ou Avaliação Relativa. A atividade de avaliação de empresas tem como propósito reunir teoria, bom-senso empresarial e vivência prática. Apesar de os modelos de avaliação de empresas serem essencialmente quantitativos, não podem ser considerados muito objetivos, pois nenhum modelo fornece um valor preciso para uma empresa e sim uma estimativa de valor (MARTELANC et al., 2005).

O objetivo do estudo é descrever as publicações que tratam do tema "Avaliação de empresas", por meio da análise de artigos publicados no Brasil. Para isso, foi desenvolvido um estudo bibliométrico com o intuito de levantar os artigos publicados em revistas brasileiras, entre 2011 e 2015, que abordaram no título, palavras-chave e/ou resumo "Avaliação de 
empresas". Em seguida foram desenvolvidas três análises específicas: as características dos periódicos, as características dos autores e as características dos artigos.

Esta pesquisa buscou responder à seguinte pergunta: quais são as características das publicações sobre avaliação de empresas, em artigos publicados nas revistas brasileiras? $\mathrm{Na}$ busca por periódicos nacionais que tratem de avaliação de empresas, observou-se a pouca quantidade de artigos abordando o assunto, dando margem a escolha do tema para esta pesquisa, contribuindo com o campo, favorecendo a futuras produções e também norteando os pesquisadores em relação ao que já foi publicado na área.

O estudo está organizado em cinco seções, iniciando com essa introdução. Posteriormente aborda-se o referencial teórico utilizado na sustentação do estudo. Em seguida é apresentada a metodologia do trabalho utilizada para desenvolver a pesquisa. Na sequência faz-se a apresentação e discussão de resultados. Ao final, apresentam-se as considerações finais do estudo realizado.

\section{REFERENCIAL TEÓRICO}

Com a finalidade de fundamentar o presente artigo, faz-se necessária a revisão da literatura sobre avaliação de empresas.

\subsection{AVALIAÇÃO DE EMPRESAS}

Para determinar o preço de uma empresa, analistas executam uma avaliação que é denominada valuation. Trata-se de uma projeção em uma estimativa do valor de uma empresa ou de alguma parte da mesma (PALEPU; HEALY; BERNARD, 2000).

O processo de avaliação de empresas consiste em alcançar seu valor justo de mercado, ou seja, aquele que representa de modo equilibrado, o potencial econômico de determinada companhia (MARTINS, 2006).

Esse valor justo também é estabelecido pelo processo de avaliação e é representado pelo valor potencial de um negócio em função de sua expectativa de geração de resultados futuros (MARTELANC; PASIN; PEREIRA, 2010).

$\mathrm{Na}$ literatura, é possível observar a existência de diversos modelos de avaliação de empresas que buscam determinar o valor das empresas. As diferenças entre esses modelos normalmente estão na abrangência das informações que são utilizadas para realizar essa estimativa e em sua aplicabilidade. Entre os mais comuns, citam-se aqueles baseados em dividendos, em valor presente dos fluxos de caixa futuros, em múltiplos de mercado e em informações contábeis (GIRÃO; MARTINS; PAULO, 2014).

$\mathrm{O}$ alto crescimento das operações de fusões e aquisições no mundo registrado nos últimos anos resulta em maior valor atribuído à atividade de avaliação de empresas e seus resultados. Disso, decorre o fato de uma das principais funções da avaliação de empresas ser: fornecer uma faixa de referência de valor da empresa que servirá como base nas negociações de fusões e aquisições (MARTELANC; TRIZI; PACHECO; PASIN, 2005).

\subsection{TÉCNICAS DE AVALIAÇÃO DE EMPRESAS}

No Brasil, o interesse e as controvérsias em relação aos métodos para determinação do valor das empresas cresceram a partir da década de 90 à medida que o processo de privatizações ganhava força e também ocorria a reestruturação do setor privado nacional por meio de fusões e incorporações (CUNHA et al., 2013).

Esses mesmos autores explicam que o debate sobre o chamado "valor justo" de uma empresa ocupou espaço na academia, assim como no meio empresarial e na imprensa, a cada 
notícia referente a uma negociação privada ou a um leilão de privatização.

O processo de avaliação de empresas teve um marco importante a partir da publicação da IN - Instrução Normativa no 361/2002 da CVM - Comissão de Valores Mobiliários. Em seu Artigo $8^{\circ}$ a Instrução estabelece que "sempre que se tratar de Oferta Pública de Aquisição de Ações - OPA formulada pela própria companhia, pelo acionista controlador ou por pessoa a ele vinculada, será elaborado laudo de avaliação da companhia objeto".

As técnicas escolhidas para avaliação de empresas devem ser coerentes com a finalidade dessa avaliação e com as especificidades da empresa avaliada para que esta possa chegar a uma conclusão que reflita o seu valor real. A aplicação equivocada de determinada técnica num processo de avaliação pode gerar resultados que não apontem a realidade e acarretar significativas perdas. As metodologias utilizadas para se avaliar empresas são: Contábil/Patrimonial; Valor de Liquidação; Múltiplos ou Avaliação Relativa; Fluxo de Caixa Descontado; EVA - Economic Value Added, entre outras. (MARTELANC et al. 2005).

Entre os modelos de avaliação destaca-se o de Ohlson (1995). A partir desse modelo, baseado na contabilidade e em outras informações que não dizem respeito a contabilidade, pesquisadores, profissionais e investidores puderam ter acesso a uma forma de avaliação diferente daquelas apresentadas até então, citadas anteriormente. Seu principal diferencial foi a abertura de novas perspectivas para melhorar a predição e a explicação dos retornos das ações (GIRÃO; MARTINS; PAULO, 2014).

Uma ampla pesquisa entre analistas de mercado revelou que os métodos mais utilizados para a avaliação são respectivamente: múltiplos de lucro, fluxo de caixa descontado e múltiplos de patrimônio (RICHARDSON; TUNA; WYSOCKI, 2010).

Na literatura especializada, o método do Fluxo de Caixa Descontado (FCD) é bastante citado e discutido. Brigham e Ehrhardt (2006) afirmam que esse método parte da premissa de que a empresa deve ser mensurada pela capacidade de gerar caixa futuro.

A avaliação corre o risco de vieses a depender de inúmeros aspectos, como por exemplo: o analista que está trabalhando nesse levantamento e as ferramentas que estão sendo utilizadas.

As fontes de viés na avaliação se iniciam com a escolha da empresa a ser avaliada. A forma de como a escolha é feita pode começar a estabelecer as bases do viés. Por exemplo, a escolha pode ter sido decorrente de uma notícia da empresa, boa ou má, que o avaliador teve acesso, iniciando uma percepção da empresa a ser avaliada. A estimativa do mercado sobre o valor da empresa reforça o viés. Avaliações que se distanciam muito do preço de mercado geram uma preocupação nos analistas que acreditam que o desvio pode refletir grandes erros de avaliação (DAMODARAN, 2007).

\section{PROCEDIMENTOS METODOLÓGICOS}

Esta pesquisa é considerada como uma bibliometria pelo modelo metodológico apresentado. Kobashi e Santos (2008, p. 109) apresentam uma definição sobre bibliometria bastante pertinente a proposta de realização deste estudo, que é:

\footnotetext{
A bibliometria é uma metodologia de recenseamento das atividades científicas e correlatas, por meio de análise de dados que apresentem as mesmas particularidades. Por meio dessa metodologia, pode-se, por exemplo, identificar a quantidade de trabalhos sobre um determinado assunto; publicados em uma data precisa; publicados por um autor ou por uma instituição ou difundidos por um periódico científico, o grau de desenvolvimento de P\&D e de inovação, entre outros. Por meios bibliométricos pode-se, por exemplo, computar dados para comparar e confrontar os elementos presentes em referências bibliográficas de documentos representativos das publicações.
}

Como são inúmeras as contribuições científicas em torno dos estudos bibliométricos, 
destaca-se nesse trabalho o artigo de Melo et al. (2013), que inspirou parte do modelo de organização e estruturação dos resultados analisados, e o estudo de Da Luz et al. (2009), que sugestionou o desenvolvimento desta pesquisa.

O procedimento técnico adotado neste estudo é classificado como de pesquisa documental, tendo em vista que os dados trabalhados foram originários dos artigos investigados e a partir daí realizaram-se análises específicas. Seguindo esse raciocínio existe a seguinte conceituação apresentada por Tozoni-Reis $(2009$, p. 30) sobre pesquisas documentais: "a busca de informações (dados) sobre os fenômenos investigados é realizado nos documentos, que exigem, para a produção de conhecimentos, uma análise".

Essa pesquisa quanto aos objetivos é considerada como descritiva, pois há um enfoque em analisar e descrever as características das publicações de periódicos nacionais envolvendo o assunto "Avaliação de Empresas". Pode-se observar o perfil desse tipo de classificação através da definição proposta por Gil (2002, p.42), que considera: "As pesquisas descritivas têm como objetivo primordial a descrição das características de determinada população ou fenômeno".

Em relação à natureza dos dados trabalhados, esta pesquisa enquadra-se como quantitativa, já que os resultados foram interpretados em números, por meio do uso da estatística descritiva, e apresentados em quadros, para auxiliar na apresentação dos resultados. Silva e Menezes (2001, p. 20) corroboram desse raciocínio e classificação ao afirmarem que: "a pesquisa quantitativa considera que tudo pode ser quantificável, o que significa traduzir em números opiniões e informações para classificá-las e analisá-las. Requer o uso de recursos e de técnicas estatísticas".

A pesquisa teve como universo de análise os artigos publicados em revistas brasileiras, inseridas no sistema QUALIS/CAPES, no período entre 2011 e 2015, e que envolviam o tema "Avaliação de Empresas".

No intuito de abranger com a maior representatividade possível artigos envolvendo o tema a ser investigado, o levantamento do universo pesquisado foi realizado a partir de buscas envolvendo cinco tipos de combinações de palavras no campo "busca avança", no Portal de Periódicos da Coordenação de Aperfeiçoamento de Pessoal de Nível Superior - CAPES. Os cinco grupos de combinações de palavras pesquisadas e que foram associadas aos campos "Qualquer" e de forma "é (exato)", foram: 1) Avaliação de Empresas and Valuation; 2) Avaliação de Empresa and Valuation; 3) Avaliação de Negócios and Valuation; 4) Avaliação Empresarial and Valuation; e 5) Avaliação de Organizações and Valuation. Como a pesquisa foi desenvolvida no campo "busca avançada", permitiu-se especificar o tipo de material, restringindo a busca apenas para "artigos", como também a data de publicação, que foi selecionada a opção "últimos 5 anos". O resultado da busca no portal, realizada em 31/ $12 / 2016$, promoveu um resultado de 20 artigos.

Após essa fase de levantamento, foram feitas algumas triagens nos 20 artigos. Primeiro, diagnosticou-se 01 artigo repetido, no grupo dos 20 materiais selecionados, reduzindo o universo para 19. Em seguida, foram observados 02 artigos publicados em periódicos estrangeiros, diminuindo o grupo de pesquisa para 17 artigos. E por fim, realizou-se uma análise em torno da abordagem dos materiais levando em consideração àqueles que continham os termos "Avaliação de Empresas" e/ou "Valuation" no título, palavras-chave ou resumo dos artigos e nisso percebeu-se que 03 publicações não se enquadravam nesse perfil. Com isso, a amostra final dessa pesquisa foi composta por 14 artigos envolvendo a temática proposta.

O estudo bibliométrico realizado permitiu que a análise dos resultados fosse dividida em três grupos: características dos periódicos, que favoreceu a descrição de peculiaridades dos periódicos que publicaram os 14 artigos; características dos autores, que permitiu analisar a formação e procedência institucional daqueles que produziram os trabalhos; e características dos artigos, que delineou o período de publicação dos mesmos, o perfil das referências 
utilizadas e as abordagens dos artigos em torno do assunto Avaliação de Empresas.

\section{ANÁLISE DOS RESULTADOS}

Os dados desta pesquisa foram obtidos a partir da análise dos 14 artigos selecionados no processo de delimitação da amostra. Assim, os resultados foram divididos em três grupos chaves que permitem descrever as características em torno das publicações científicas brasileiras envolvendo o tema avaliação de empresas, entre 2011 e 2015. Com isso, seguem as análises dos dados em cada um dos grupos.

\subsection{CARACTERÍSTICAS DOS PERIÓDICOS}

Neste grupo serão apresentados três quadros com informações sobre os periódicos que contém os artigos analisados. Assim, seguem os quadros com as suas respectivas análises.

O Quadro 01 descreve os periódicos em que os 14 artigos selecionados foram publicados, a frequência de publicação por revista e a classificação promovida pela Coordenação de Aperfeiçoamento de Pessoal de Nível Superior - CAPES aos periódicos investigados.

\section{Quadro 01 - Classificação dos periódicos}

\begin{tabular}{|l|c|c|c|}
\hline \multicolumn{1}{|c|}{ Periódico } & ISSN & Classificação & Frequência \\
\hline Revista de Administração & $0080-2107$ & A2 & 2 \\
\hline Revista de Administração Mackenzie & $1518-6776$ & B1 & 2 \\
\hline Revista Brasileira de Finanças & $1679-0731$ & B1 & 2 \\
\hline Gestão \& Produção & $0104-530 X$ & B1 & 1 \\
\hline Revista de Gestão - REGE & $1809-2276$ & B1 & 1 \\
\hline Revista de Contabilidade e Organizações & $1982-6486$ & B1 & 1 \\
\hline Revista Ambiente Contabil & $2176-9036$ & B3 & 1 \\
\hline Revista Catarinense da Cência Contábil & $1808-3781$ & B3 & 1 \\
\hline Revista de Contabilidade \& Controladoria & $1984-6266$ & B3 & 1 \\
\hline Revista de Gestão e Projetos & $2236-0972$ & B3 & 1 \\
\hline Revista de Gestão, Finanças e Contabilidade & $2238-5320$ & B3 & $\mathbf{1 4}$ \\
\hline \multicolumn{2}{|c|}{ Total de Publicações } & & 1 \\
\hline
\end{tabular}

Fonte: Dados da Pesquisa, 2016

Percebe-se que três periódicos se destacaram por possuírem uma frequência maior de artigos no período estudado, que foram: a Revista de Administração; a Revista de Administração Mackenzie; e a Revista Brasileira de Finanças.

Outro ponto observado no Quadro 01 é a classificação das revistas pelo conceito CAPES. Tal conceituação foi extraída da plataforma Sucupira, através da busca no campo "Classificação de periódicos 2015" e levando em consideração a área de avaliação "Administração, Ciências Contábeis e Turismo" em todas as revistas. Sendo assim, evidenciase que o estrato com maior número de publicações é o B1 com um total de 07 publicações, ou seja, 50\% do universo analisado. Destacam-se também duas publicações no grupo A2, que pertencem a Revista de Administração.

No Quadro 02 são descritas as instituições que estão vinculadas aos 11 periódicos, como também as cidades onde estão localizadas as mesmas. 
Quadro 02 - Instituição e localização dos periódicos

\begin{tabular}{|l|c|c|c|}
\hline \multicolumn{1}{|c|}{ Periódico } & Instituição & Localização & Frequência \\
\hline Revista de Administração & FEA/USP & São Paulo/SP & 2 \\
\hline Revista de Administração Mackenzie & U. P. Mackenzie & São Paulo/SP & 2 \\
\hline Revista Brasileira de Finanças & SBFin & Rio de Janeiro/RJ & 2 \\
\hline Gestão \& Produção & UFSCAR & São Carlos/SP & 1 \\
\hline Revista de Gestão - REGE & FEA/USP & São Paulo/SP & 1 \\
\hline Revista de Contabilidade e Organizações & FEA/USP & São Paulo/SP & 1 \\
\hline Revista Ambiente Contábil & UFRN & Natal/RN & 1 \\
\hline Revista Catarinense da Ciência Contábil & CRC - SC & Florianópolis/SC & 1 \\
\hline Revista de Contabilidade \& Controladoria & UFPR & Curitiba/PR & 1 \\
\hline Revista de Gestão e Projetos & UNINOVE & São Paulo/SP & 1 \\
\hline Revista de Gestão, Finanças e Contabilidade & UNEB & Salvador/BA & 1 \\
\hline \multicolumn{2}{|c|}{ Total de Publicações } & & $\mathbf{1 4}$ \\
\hline
\end{tabular}

Fonte: Dados da Pesquisa, 2016

Com relação às instituições, evidencia-se que a Faculdade de Administração, Economia e Contabilidade - FEA, da Universidade de São Paulo - USP, apresenta nos dados do Quadro 02 três periódicos distintos que publicaram sobre o tema em análise, totalizando 04 artigos.

No quesito distribuição dos artigos por cidades das instituições, percebe-se uma elevada concentração de publicações na cidade de São Paulo, com um somatório de 07 trabalhos, o que indica $50 \%$ do universo estudado.

A seguir tem-se o Quadro 03 que apresenta a distribuição regionalizada das instituições que publicaram sobre o tema.

Quadro 03 - Distribuição regionalizada dos periódicos analisados

\begin{tabular}{|c|c|c|c|}
\hline Cidade/Estado & Região & $\begin{array}{c}\text { Quant. de } \\
\text { Publicações }\end{array}$ & Percentual (\%) por Região \\
\hline Natal/RN & Nordeste & 1 & \multirow{2}{*}{14,286} \\
\hline Salvador/BA & Nordeste & 1 & \\
\hline Rio de Janeiro/RJ & Sudeste & 2 & \multirow{3}{*}{71,429} \\
\hline São Carlos/SP & Sudeste & 1 & \\
\hline São Paulo/SP & Sudeste & 7 & \\
\hline Curitiba/PR & Sul & 1 & \multirow{2}{*}{14,286} \\
\hline Florianópolis/SC & Sul & 1 & \\
\hline \multicolumn{2}{|c|}{ Totais } & 14 & 100,000 \\
\hline
\end{tabular}

Fonte: Dados da Pesquisa, 2016

Há uma evidenciação da representatividade do número de publicações que a região Sudeste possui em relação às demais regiões, totalizando 10 artigos, aproximadamente $71,43 \%$ do universo. Em seguida tem-se o Nordeste e o Sul com 02 publicações cada. Percebe-se que, diante dos traços do ambiente estudado, o Norte e o Centro-Oeste não apresentam periódicos com trabalhos envolvendo o tema.

Sendo assim, ao analisar as Características dos Periódicos, existem alguns pontos importantes a serem considerados em relação à classificação dos periódicos e a localização dos mesmos.

No quesito classificação das revistas no Conceito CAPES, aparecem três tipos de GєCont, v. 4, n. 1, Floriano-PI, Jan-Jun. 2017. 
estratos distintos (A2, B1 e B3), sendo os periódicos de classificação B1 que concentram o maior volume de publicações.

Já no âmbito da distribuição geográfica, a região Sudeste destaca-se como a principal divulgadora dos trabalhos sobe o tema, com ênfase para a cidade de São Paulo.

\subsection{CARACTERÍSTICAS DOS AUTORES}

No estudo realizado foram utilizados 14 artigos que somaram o total de 39 autores, no entanto 4 autores participaram de mais de um artigo, por isso foram analisados nesse tópico 33 pesquisadores distintos.

O Quadro 4 representa a distribuição da quantidade de autores por artigo elaborado. Ao analisar essa distribuição, percebe-se que 7 artigos (50,00\% da amostra) possuem dois autores na elaboração do estudo. Observa-se também que existem 04 artigos elaborados com a participação de quatro autores, o que caracteriza $28,57 \%$ do universo.

Quadro 4 - Quantidade de autores por artigo.

\begin{tabular}{|l|c|c|c|c|}
\hline Quant. Autores por artigo & 2 autores & 3 autores & 4 autores & Total \\
\hline Frequência de artigos & 7 & 3 & 4 & $\mathbf{1 4}$ \\
\hline Percentual (\%) & $50 \%$ & $21,43 \%$ & $28,57 \%$ & $\mathbf{1 0 0 \%}$ \\
\hline
\end{tabular}

Fonte: Dados da pesquisa, 2016.

O Quadro 5 apresenta a titulação máxima dos pesquisadores envolvidos ou o estágio de formação do autor no momento da elaboração do artigo, levando em consideração até a titulação de doutorado.

Enfatiza-se que os dados sobre os níveis e a área de formação dos pesquisadores foram obtidos nas próprias informações divulgadas nos artigos ou, quando os artigos não possuíam os detalhes específicos, foi consultado o Currículo Lattes de tais autores (observando o período associado à publicação dos artigos).

Ao avaliar os dados do Quadro 5, percebe-se uma grande concentração de doutores envolvidos nos artigos selecionados, representando $63,64 \%$ do universo. Outro ponto observado é a diversidade de áreas de formação dos autores envolvidos na pesquisa sobre avaliação de empresas: Administração, Economia, engenharia de produção, entre outras.

Quadro 5 - Titulação ou estágio de Formação dos pesquisadores.

\begin{tabular}{|c|c|c|}
\hline Titulação ou estágio de formação & Quant. de pesquisadores & Frequência \% por grupo \\
\hline Doutor em Administração & 6 & \multirow{5}{*}{$63,64 \%$} \\
\hline Doutor em Ciências Contábeis & 5 & \\
\hline Doutor em Controladoria e Contabilidade & 4 & \\
\hline Doutor em Economia & 3 & \\
\hline Doutor em Engenharia de Produção & 3 & \\
\hline Doutorando em Contabilidade & 1 & $3,03 \%$ \\
\hline Mestre em Administração & 2 & \multirow{5}{*}{$21,21 \%$} \\
\hline Mestre em Agronegócio & 1 & \\
\hline Mestre em Ciências Contábeis & 3 & \\
\hline Mestre em Contabilidade e Finanças & 1 & \\
\hline Mestre em Economia & 1 & \\
\hline Graduação em Ciências Contábeis & 2 & \multirow[t]{2}{*}{$12,12 \%$} \\
\hline Graduação em Direito & 1 & \\
\hline Total & 33 & $100 \%$ \\
\hline
\end{tabular}

Fonte: Dados da pesquisa, 2016.

Foi possível observar que 16 dos autores estão envolvidos na área das Ciências Contábeis, representando $48,48 \%$ do total da amostra.

GєCont, v. 4, n. 1, Floriano-PI, Jan-Jun. 2017. 
No Quadro 6 ocorre a distribuição por localização dos autores, de acordo com o as instituições em que os mesmos estavam vinculados no período de elaboração dos artigos.

Quadro 6 - Instituição vinculada ao Pesquisador

\begin{tabular}{|l|c|c|}
\hline \multicolumn{1}{|c|}{ Instituição vinculada ao pesquisador } & $\begin{array}{c}\text { Quant. de } \\
\text { pesquisadores }\end{array}$ & $\begin{array}{c}\text { Frequência \% } \\
\text { por grupo }\end{array}$ \\
\hline Companhia Energética de Goiás - CELG & 1 & $3,03 \%$ \\
\hline Fac. de Econ., Adm. e Cont. da Univ. de São Paulo - FEA USP & 3 & $9,09 \%$ \\
\hline Fund. Inst. de Pesq. Cont., Atuariais e Financeiras - FIPECAFI. & 1 & $3,03 \%$ \\
\hline Fundação Pedro Leopoldo - FPL & 3 & $9,09 \%$ \\
\hline Fund. Inst. Capixaba de Pesq. em Cont., Econ. e Finanças - FUCAPE. & 2 & $6,06 \%$ \\
\hline Inst. Federal de Educação, Ciência e Tecnologia do RN - IFRN. & 1 & $3,03 \%$ \\
\hline Private Equity da Rio Bravo Investimentos. & 1 & $3,03 \%$ \\
\hline Pontifícia Universidade Católica de Goiás - PUC Goiás & 1 & $3,03 \%$ \\
\hline Pontifícia Universidade Católica do Rio de Janeiro - PUC Rio & 1 & $3,03 \%$ \\
\hline Universidade Federal do Espírito Santo - UFES & 2 & $6,06 \%$ \\
\hline Universidade Federal de Goiás - UFG & 5 & $15,16 \%$ \\
\hline Universidade Federal da Paraíba - UFPB & 2 & $6,06 \%$ \\
\hline Universidade Federal de Pernambuco - UFPE & 2 & $6,06 \%$ \\
\hline Universidade Federal do Rio de Janeiro - UFRJ & 3 & $9,09 \%$ \\
\hline Universidade Federal do Rio Grande do Norte - UFRN & 1 & $3,03 \%$ \\
\hline Universidade Federal Rural de Pernambuco - UFRPE & 1 & $3,03 \%$ \\
\hline Universidade Federal de Santa Catarina - UFSC & 1 & $3,03 \%$ \\
\hline Universidade Federal de Uberlândia - UFU & 1 & $3,03 \%$ \\
\hline Universidade de Brasília - UNB & 1 & $3,03 \%$ \\
\hline \multicolumn{1}{c|}{ Total } & $\mathbf{3 3}$ & $\mathbf{1 0 0 \%}$ \\
\hline
\end{tabular}

Fonte: Dados da pesquisa, 2016.

Ainda no Quadro 6, percebem-se quatro instituições com a maior frequência de pesquisadores atuantes no estudo sobre avaliação de empresas, que são: UFG - Universidade Federal de Goiás; FEA USP - Fac. de Econ., Adm. e Cont. da Univ. de São Paulo; FPL Fundação Pedro Leopoldo; e UFRJ - Universidade Federal do Rio de Janeiro.

Apesar da maioria dos autores estarem relacionados a instituições de ensino, observa-se a existência de pesquisadores vinculados a outros tipos de instituições, como: a CELG Companhia Energética de Goiás e a Private Equity da Rio Bravo Investimentos.

No Quadro 07 observa-se a distribuição dos autores nos estados brasileiros, através da localização institucional em que eles estavam vinculados no período de elaboração dos estudos, permitindo observar a disposição dos mesmos por região brasileira.

Observa-se que os estados de Goiás e São Paulo possuem o maior volume de pesquisadores atuantes na área analisada, com um quantitativo de 12 autores, representando um percentual aproximado de $36,36 \% \%$ do total de pesquisadores. 
Quadro 7 - Localização Institucional dos autores

\begin{tabular}{|c|c|c|c|}
\hline Estado & Região & $\begin{array}{l}\text { Quant. de } \\
\text { autores }\end{array}$ & 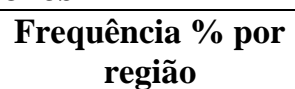 \\
\hline Distrito Federal & Centro-Oeste & 1 & \multirow{2}{*}{$24,24 \%$} \\
\hline Goiás & Centro-Oeste & 7 & \\
\hline Minas Gerais & Sudeste & 4 & \multirow{4}{*}{$51,52 \%$} \\
\hline Espírito Santo & Sudeste & 4 & \\
\hline Rio de Janeiro & Sudeste & 4 & \\
\hline São Paulo & Sudeste & 5 & \\
\hline Santa Catarina & Sul & 1 & $3,03 \%$ \\
\hline Pernambuco & Nordeste & 3 & \multirow{3}{*}{$21,21 \%$} \\
\hline Rio Grande do Norte & Nordeste & 2 & \\
\hline Paraíba & Nordeste & 2 & \\
\hline Total & & 33 & $100 \%$ \\
\hline
\end{tabular}

Fonte: Dados da pesquisa, 2016.

Com relação à visualização da disposição dos autores por região brasileira, a região Sudeste concentra o maior número autores, com um percentual de 51,52\% do universo de autores. Já a região Centro-Oeste aparece em segundo lugar em relação ao maior volume de autores, com $24,24 \%$.

Um ponto de destaque é a ausência de autores da região Norte e a pouca representatividade da região Sul, com apenas um pesquisador, representando 3,03\%.

$\mathrm{Na}$ análise das características dos autores, percebeu-se que nenhum artigo foi escrito individualmente, tendo entre dois e quatro autores por artigo. Observou-se uma predominância de doutores envolvidos no processo de pesquisa, representando 63,64\% do universo. Destacase também a elevada participação de profissionais ligados área contábil e a grande participação de autores da região Sudeste.

\subsection{CARACTERÍSTICAS DOS ARTIGOS}

Neste tópico serão explorados 08 quadros que envolverão os detalhes dos materiais avaliados como: títulos; objetivos; ano de publicação; e referências.

Para iniciar a análise das características dos artigos, no Quadro 08 evidenciaram-se os títulos e autores dos artigos estudados. Percebe-se que foram relacionados números para cada um dos 14 materiais. Tal numeração foi estabelecida no intuito de facilitar a classificação dos objetivos que serão apresentados no Quadro 09. 
Quadro 08 - Títulos e autores das pesquisas analisadas.

\begin{tabular}{|c|c|c|}
\hline Artigo & $\begin{array}{l}\text { Título } \\
\end{array}$ & Autores \\
\hline 01 & $\begin{array}{l}\text { Avaliação de empresas: uma análise sob a óptica do "viés de } \\
\text { avaliação" nos laudos de oferta pública de aquisição de ações }\end{array}$ & Santos e Cunha (2015) \\
\hline 02 & $\begin{array}{l}\text { Os analistas sell-side fazem boas previsões de preços-alvo no } \\
\text { Brasil? }\end{array}$ & Lima e Almeida (2015) \\
\hline 03 & $\begin{array}{l}\text { Avaliação e gestão econômico-financeira de projetos: um estudo } \\
\text { aplicado ao setor metalúrgico }\end{array}$ & $\begin{array}{l}\text { Moreira, Locatelli e Afonso } \\
\text { (2015) }\end{array}$ \\
\hline 04 & $\begin{array}{l}\text { Avaliação de empresas e probabilidade de negociação com } \\
\text { informação privilegiada no mercado brasileiro de capitais }\end{array}$ & $\begin{array}{c}\text { Girão, Martins e Paulo } \\
\text { (2014) }\end{array}$ \\
\hline 05 & $\begin{array}{l}\text { Avaliação de empresas no Brasil pelo fluxo de caixa descontado: } \\
\text { evidências empíricas sob o ponto de vista dos direcionadores de } \\
\text { valor nas ofertas públicas de aquisição de ações }\end{array}$ & $\begin{array}{l}\text { Cunha, Martins e Assaf Neto } \\
\text { (2014) }\end{array}$ \\
\hline 06 & $\begin{array}{l}\text { Avaliação de empresas start-up por Opções Reais: o caso do setor } \\
\text { de biotecnologia }\end{array}$ & Rodrigues et al. (2013) \\
\hline 07 & $\begin{array}{l}\text { Custo de capital médio ponderado na avaliação de empresas no } \\
\text { Brasil: uma investigação da aderência acadêmica e a prática de } \\
\text { mercado }\end{array}$ & Cunha et al. (2013) \\
\hline 08 & $\begin{array}{l}\text { Análise das alíquotas de IR e CS usadas nos laudos de avaliação de } \\
\text { empresas brasileiras, nos casos de oferta pública de aquisição de } \\
\text { ações }\end{array}$ & Cunha et al. (2013) \\
\hline 09 & $\begin{array}{l}\text { Apuração de haveres de sócio: o caso de uma empresa do comércio } \\
\text { varejista da grande Florianópolis }\end{array}$ & Silva e Santos (2013) \\
\hline 10 & $\begin{array}{l}\text { Avaliação de empresas por múltiplos aplicados em empresas } \\
\text { agrupadas com análise de cluster }\end{array}$ & Couto Júnior e Galdi (2012) \\
\hline 11 & Simulação de Monte Carlo e valuation: uma abordagem estocástica & $\begin{array}{l}\text { Oliveira e Medeiros Neto } \\
\text { (2012) }\end{array}$ \\
\hline 12 & $\begin{array}{l}\text { Análise dos modelos de avaliação Residual Income Valuation, } \\
\text { Abnormal Earnings Growth e Fluxo de Caixa Descontado aplicados } \\
\text { às ofertas públicas de aquisição de ações no Brasil }\end{array}$ & Almeida et al. (2012) \\
\hline 13 & $\begin{array}{l}\text { Avaliação de ações e números contábeis: aplicação dos modelos } \\
\text { Zhang (2000) e Zhang }\end{array}$ & Galdi e Lopes (2011) \\
\hline 14 & $\begin{array}{l}\text { Determinantes da formação do capital intelectual nas empresas } \\
\text { produtoras de tecnologia da informação e comunicação }\end{array}$ & Lima e Carmona (2011) \\
\hline
\end{tabular}

Fonte: Dados da Pesquisa, 2016.

As contribuições dos 14 artigos investigados em torno da temática podem ser percebidas através do Quadro 09, que demonstra os enfoques dos objetivos desses 14 artigos. Para a construção deste quadro foi necessária a avaliação dos objetivos, das palavras-chave e do conteúdo dos artigos. Após tal avaliação, percebeu-se que existiam objetivos envolvendo apenas um enfoque de estudo, como também havia outros com mais de um enfoque. Assim, no Quadro 09, foram expostas 10 categorias de enfoques identificados nos artigos avaliados, que são: modelos de avaliação de empresa, que envolve estudos teóricos ou aplicação de tais modelos em casos concretos; oferta pública de aquisição de ação - OPA; laudos de avaliação de empresa; direcionadores de valor; retornos de ações; avaliação de intangíveis; assimetria de informação na avaliação de empresas; previsão de preços de ações; avaliação de projetos; e apuração de haveres de sócios. 
Quadro 09 - Enfoques dos objetivos dos artigos pesquisados

\begin{tabular}{|l|c|c|c|}
\hline \multicolumn{1}{|c|}{$\begin{array}{c}\text { Enfoques dos objetivos dos artigos } \\
\text { pesquisados }\end{array}$} & $\begin{array}{c}\text { Artigos } \\
\text { relacionados }\end{array}$ & $\begin{array}{c}\text { Frequência do } \\
\text { enfoque } \\
\text { abordado }\end{array}$ & $\begin{array}{c}\text { Percentual de participação } \\
\text { dos enfoques em relação ao } \\
\text { universo dos 14 artigos } \\
\text { estudados }\end{array}$ \\
\hline Modelos de avaliação de empresa & $\begin{array}{c}03 ; 04 ; 06 ; 07 ; \\
08 ; 10 ; 11 ; 12 ; \\
13 ; 14\end{array}$ & 10 & 71,43 \\
\hline Oferta pública de aquisição de ação & $01 ; 07 ; 08 ; 12$ & 4 & 28,57 \\
\hline Laudos de avaliação & $01 ; 05 ; 08 ; 12$ & 4 & 28,57 \\
\hline Direcionadores de valor & $05 ; 14$ & 2 & 14,29 \\
\hline Retornos de ações & $02 ; 13$ & 2 & 14,29 \\
\hline Avaliação de intangíveis & $09 ; 14$ & 2 & 14,29 \\
\hline $\begin{array}{l}\text { Assimetria de informação na avaliação de } \\
\text { empresas }\end{array}$ & 04 & 1 & 7,15 \\
\hline Previsão de preços de ações & 02 & 1 & 7,15 \\
\hline Avaliação de projetos & 03 & 1 & 7,15 \\
\hline Apuração de haveres de sócios & 09 & 1 & 7,15 \\
\hline
\end{tabular}

Fonte: Dados da Pesquisa, 2016.

Nisso, percebe-se que o enfoque mais abordado envolveu modelos de avaliação de empresa, totalizando 10 artigos, que representa $71,43 \%$ do universo. As categorias de oferta pública de aquisição de ação e laudos de avaliação ficaram na segunda posição dos enfoques adotados, com um envolvimento cada um em 04 objetivos, ou seja, 28,57\% da amostra. Em seguida surgiram os enfoques que trabalham com direcionadores de valor, retornos de ações e avaliação de intangíveis, que se relacionaram cada um desses com 02 artigos. Por fim, têm-se os grupos de assimetria de informação na avaliação de empresas, previsão de preços de ações, avaliação de projetos e apuração de haveres de sócio, que se apresentaram apenas uma vez nos objetivos analisados, cada um respectivamente.

O Quadro 10 demonstra a frequência de publicações dos artigos ao longo do período entre 2011 e 2015.

Quadro 10 - Distribuição das publicações por ano.

\begin{tabular}{|l|c|c|c|c|c|c|}
\hline \multicolumn{1}{|c|}{ Ano das Publicações } & $\mathbf{2 0 1 1}$ & $\mathbf{2 0 1 2}$ & $\mathbf{2 0 1 3}$ & $\mathbf{2 0 1 4}$ & $\mathbf{2 0 1 5}$ & Total \\
\hline Quantidade de Publicações & 2 & 3 & 4 & 2 & 3 & $\mathbf{1 4}$ \\
\hline Percentual (\%) & 14,29 & 21,43 & 28,57 & 14,29 & 21,43 & $\mathbf{1 0 0 , 0 0}$ \\
\hline
\end{tabular}

Fonte: Dados da Pesquisa, 2016.

O ano que se destacou com um maior número de publicações foi o de 2013, totalizando 04 artigos (28,57 \% da amostra). Percebe-se que 2013 foi o ápice da crescente do número de publicações envolvendo o triênio de 2011 a 2013, logo após ocorreu um decréscimo (2014), com uma nova evolução em 2015.

Pode-se verificar o número total de referências utilizadas nos artigos, bem como as suas origens, através do Quadro 11 abaixo. 
Quadro 11 - Procedência das Referências.

\begin{tabular}{|c|c|c|}
\hline \multicolumn{1}{|c|}{ Tipo de Publicação } & Número de Referências & Percentual (\%) \\
\hline Nacional & 178 & 41,98 \\
\hline Internacional & 246 & 58,02 \\
\hline Total & $\mathbf{4 2 4}$ & $\mathbf{1 0 0 , 0 0}$ \\
\hline
\end{tabular}

Fonte: Dados da Pesquisa, 2016.

Assim, nos 14 materiais foram utilizadas 424 referências, sendo que as produções estrangeiras possuíram uma maior evidência, tendo em vista um montante de aplicação de 246 obras (58,02\% do universo), enquanto as referências nacionais somaram 178 unidades (41,98\% do total).

O Quadro 12 detalha os tipos de fontes referenciados mais de uma vez, ou seja, nessa análise consideraram-se as produções que tinham a mesma autoria, título, origem documental e que foram citados pelo menos duas vezes nas referências do universo dos 14 artigos investigados. Sendo assim, observou-se um total de 104 referências com essas características.

Diante dos dados apresentados no Quadro 12, os livros de publicação nacional apresentaram maior evidência com uma participação de 43 referências $(41,35 \%$ do universo de 104 materiais).

Quadro 12 - Distribuição dos documentos referenciados mais de uma vez

\begin{tabular}{|c|c|c|c|c|c|c|}
\hline \multirow{2}{*}{$\begin{array}{c}\text { Fonte de documentos } \\
\text { referenciados mais de uma } \\
\text { vez }\end{array}$} & \multicolumn{2}{|c|}{ Nacional } & \multicolumn{2}{|c|}{ Internacional } & \multicolumn{2}{|c|}{ Total } \\
\hline & Frequência & $\%$ & Frequência & $\%$ & Frequência & $\%$ \\
\hline Livros & 43 & 41,35 & 16 & 15,38 & 59 & 56,73 \\
\hline Periódicos & 4 & 3,85 & 29 & 27,88 & 33 & 31,73 \\
\hline Anais de Congresso & 8 & 7,69 & 0 & 0,00 & 8 & 7,69 \\
\hline Outros & 4 & 3,85 & 0 & 0,00 & 4 & $\mathbf{3 , 8 5}$ \\
\hline Total & 59 & 56,73 & 45 & 43,27 & 104 & 100,00 \\
\hline
\end{tabular}

Fonte: Dados da Pesquisa, 2016.

O segundo grupo mais utilizado foi o do periódico internacional, representando um total de 29 artigos (27,88\% dos 104 itens). Em sequência, em ordem de volume, surgiram os seguintes tipos de referências utilizados mais de uma vez: livros internacionais, atuando com $15,38 \%$ do grupo; anais de congresso nacional, participando com 7,69\%; periódicos nacionais, que representou $3,85 \%$; e outros, formado por uma norma e uma tese, que também atuou com $3,85 \%$.

No Quadro 13 está disponível a distribuição dos 12 periódicos que tiveram artigos referenciados mais de uma vez. 
Quadro 13 - Periódicos com artigo referenciados mais de uma vez.

\begin{tabular}{|l|c|c|}
\hline \multicolumn{1}{|c|}{ Periódico com artigo referenciado mais de uma vez } & Origem & Frequência \\
\hline The Journal of Finance & Internacional & 6 \\
\hline Contemporary Accounting Research & Internacional & 5 \\
\hline American Economic Review & Internacional & 3 \\
\hline Journal of Financial and Quantitative Analysis & Internacional & 3 \\
\hline Accounting Horizons & Internacional & 2 \\
\hline Forthcoming in the Journal of Financial Economics & Internacional & 2 \\
\hline Journal of Accounting and Economics & Internacional & 2 \\
\hline Harvard Business Review & Internacional & 2 \\
\hline American Economic Review & Internacional & 2 \\
\hline Review of Financial Studies & Internacional & 2 \\
\hline Revista de Contabilidade e Finanças & Nacional & 2 \\
\hline Unb Contábil & Nacional & 2 \\
\hline \multicolumn{2}{|c}{ Total } & $\mathbf{3 3}$ \\
\hline
\end{tabular}

Fonte: Dados da Pesquisa, 2016.

No quadro apresentado, os dois periódicos mais utilizados tiveram origem estrangeira, que foram: The Journal of Finance; e o Contemporary Accounting Research. Já a "Revista de Contabilidade e Finanças" e a "Unb Contábil" foram os periódicos brasileiros que tiveram artigos referenciados mais de uma vez.

Como último ponto a ser analisado, tem-se o Quadro 14, que expõe os títulos dos materiais mais citados, bem como os respectivos autores responsáveis por tais obras.

No Quadro 14 foram apresentadas todas as referências citadas pelo menos três vezes e que formam um grupo de 08 livros e 04 artigos. Observa-se que os três materiais mais citados são livros de edição nacional, com destaque para o livro organizado por Eliseu Martins, que foi utilizado 07 vezes, ou seja, do universo de 14 artigos estudados, tal obra serviu de referência para 50\% dos materiais. Outro ponto importante evidenciado foi a participação do autor Aswath Damodaran que apareceu com três livros distintos no rol das referências mais utilizadas. 
Quadro 14 - Referências mais utilizadas

\begin{tabular}{|c|c|c|c|}
\hline Tipo & Título & Autores & Freq. \\
\hline Livro & $\begin{array}{l}\text { Avaliação de empresas: da mensuração contábil à } \\
\text { econômica. }\end{array}$ & $\begin{array}{l}\text { MARTINS, Eliseu } \\
\text { (Org.) }\end{array}$ & 07 \\
\hline Livro & $\begin{array}{l}\text { Avaliação de empresas - valuation: calculando e } \\
\text { gerenciando o valor das empresas. }\end{array}$ & $\begin{array}{l}\text { COPELAND, Tom; } \\
\text { KOLLER, Tim; } \\
\text { MURRIN, Jack. }\end{array}$ & 06 \\
\hline Livro & Avaliação de empresas. & $\begin{array}{l}\text { DAMODARAN, } \\
\text { Aswath }\end{array}$ & 05 \\
\hline Livro & $\begin{array}{l}\text { Valuation approaches and metrics: a survey of the } \\
\text { theory and evidence. }\end{array}$ & $\begin{array}{l}\text { DAMODARAN, } \\
\text { Aswath } \\
\end{array}$ & 04 \\
\hline Livro & $\begin{array}{l}\text { Business analysis \& valuation: using financial } \\
\text { statements. }\end{array}$ & $\begin{array}{l}\text { PALEPU, Krishna G.; } \\
\text { HEALY, Paul M.; } \\
\text { BERNARD, Victor L. }\end{array}$ & 04 \\
\hline Artigo & $\begin{array}{l}\text { Interactions of corporate financing and investment } \\
\text { decisions-implications for capital budgeting. }\end{array}$ & MYERS, Stewart. & 04 \\
\hline Livro & Valuationmethodsandshareholdervaluecreation & FERNÁNDEZ, Pablo. & $\mathbf{0 3}$ \\
\hline Livro & $\begin{array}{l}\text { Avaliação de investimentos: ferramentas e técnicas para } \\
\text { a determinação do valor de qualquer ativo. }\end{array}$ & $\begin{array}{l}\text { DAMODARAN, } \\
\text { Aswath }\end{array}$ & 03 \\
\hline Livro & Análise estatística com o PaswStatistics & MARÔCO, João & $\mathbf{0 3}$ \\
\hline Artigo & $\begin{array}{l}\text { The weighted average cost of capital, perfect capital } \\
\text { markets and Project life: a clarification. }\end{array}$ & $\begin{array}{l}\text { MILES, James. A.; } \\
\text { EZZELL, John. R. }\end{array}$ & $\mathbf{0 3}$ \\
\hline Artigo & $\begin{array}{l}\text { The cost of capital, corporation finance and the theory } \\
\text { of investment. }\end{array}$ & $\begin{array}{l}\text { MODIGLIANI, Franco; } \\
\text { MILLER, Merton H. }\end{array}$ & $\mathbf{0 3}$ \\
\hline Artigo & $\begin{array}{l}\text { Earnings, book values, and dividends in equity } \\
\text { valuation. }\end{array}$ & Ohlson, James. & $\mathbf{0 3}$ \\
\hline
\end{tabular}

Fonte: Dados da Pesquisa, 2016.

A análise das características dos artigos permitiu evidenciar que a grande maioria dos objetivos analisados envolve estudos teóricos e/ou aplicação concreta de modelos de avaliação de empresas. Ao longo da linha temporal pesquisada, o ano que mais se publicou sobre o assunto foi o de 2013, que totalizou 04 artigos, 28,57\% do universo.

Com relação ao perfil das referências utilizadas, percebe-se uma predominância do uso de materiais internacionais, conforme observado no Quadro 11. Já na análise detalhada das fontes das referências utilizadas mais de uma vez, percebe-se que os Livros nacionais foram os mais utilizados.

Outro ponto observado na análise das referências é a lista de periódicos que tiveram artigos citados mais de uma vez. Nesse quesito, percebe-se uma elevada concentração de periódicos internacionais utilizados, conforme Quadro 13.

\section{CONSIDERAÇÕES FINAIS}

As informações apresentadas nos três grupos de informações propostos (periódicos, autores e artigos) envolvendo os dados do universo dessa pesquisa, permitiram promover a análise e descrição das características em torno das publicações científicas brasileiras envolvendo o tema "Avaliação de Empresas", objetivo deste estudo.

Com relação às características dos periódicos que publicaram os 14 artigos estudados percebe-se uma predominância de publicações no estrato B1, no conceito de qualificação CAPES. Já os periódicos que mais publicaram no período avaliado são: a Revista de Administração, da FEA/USP; a Revista de Administração Mackenzie, da Universidade Presbiteriana Mackenzie; e a Revista Brasileira de Finanças, que está vinculada a Sociedade Brasileira de Finanças. Na análise geográfica dos periódicos, percebe-se a expressiva concentração de publicações na região Sudeste, com ênfase para a cidade de São Paulo, que foi responsável por $50 \%$ das publicações.

GєCont, v. 4, n. 1, Floriano-PI, Jan-Jun. 2017. 
No grupo de análises sobre as características dos autores, a produção do artigo em dupla se apresentou como o tipo predominante. Já no quesito Titulação ou Estágio de Formação dos pesquisadores, evidenciaram-se dois fatos: 1) a grande participação de Doutores em torno da temática; 2) e o elevado número de profissionais ligados a contabilidade envolvidos com a pesquisa na área de Avaliação de Empresas, representando 48,48\% dos autores. A análise das instituições que estão envolvidas com os pesquisadores demonstra um equilíbrio percentual de participação, com destaque para a Universidade Federal de Goiás - UFG que apresentou o maior número de autores. Percebeu-se também o envolvimento de 02 profissionais não vinculados a universidades, mas relacionados com as seguintes empresas: Companhia Energética de Goiás; e a Private Equity da Rio Bravo Investimentos. Na análise regionalizada dos autores evidencia-se a elevada participação de pesquisadores do Sudeste, com uma participação de $51,52 \%$ do grupo. No entanto, em comparação aos locais de publicação, vale ressaltar o envolvimento de pesquisadores da região Centro-Oeste, com 24,24\% dos autores e o baixo número da região Sul, com apenas 01 autor. Tais números demonstram uma migração de informação, ou seja, há certa mobilidade entre o local que se produz e o ambiente que se publica.

No âmbito das características dos artigos, evidenciou-se na maioria das pesquisas a vinculação dos objetivos propostos com estudos envolvendo modelos de avaliação de empresas, segundo os dados apresentados no Quadro 08. Já na linha temporal definida para essa pesquisa, o ano de 2013 se destacou como àquele responsável pelo maior número de publicações. No quesito procedência das referências utilizadas para embasar a construção dos artigos, em números totais, as referências estrangeiras tiveram uma maior participação comparando-se com as nacionais. $\mathrm{Na}$ análise das citações dos materiais que foram referenciados pelo menos duas vezes, percebe-se que os livros nacionais foram as fontes mais utilizadas. No entanto, ainda nesse grupo de resultado, ficou evidenciado que os periódicos internacionais ficaram na segunda posição em volume de frequência de utilização, com destaque para o The Journal of Finance e o Contemporary Accounting Research. E por fim, tem-se, na análise das referências mais utilizadas, o livro "Avaliação de empresas: da mensuração contábil à econômica", organizado pelo autor Eliseu Martins, como a obra mais contemplada para embasamento teórico e o autor Aswath Damodaran com três livros no rol das bibliografias que tiveram maior frequência de consulta.

Diante do exposto, os resultados apresentados nesse estudo, envolvendo os três grupos de características analisadas, irão contribuir diretamente com futuras pesquisas que desenvolverem trabalhos na área de "Avaliação de Empresas". Já que as informações apresentadas poderão nortear a construção e a publicação de futuros estudos em torno dessa temática. Este estudo também pode favorecer o processo de decisão e planejamento de instituições vinculadas à edição de periódicos com interesse em publicação na área.

Como limitação do estudo destaca-se a abrangência da investigação em uma única base de dados. Por isso, sugere-se para futuras pesquisas, envolvendo estudos bibliométricos sobre "Avaliação de Empresas", a utilização de outras bases de dados, ou até mesmo o próprio portal de periódicos da CAPES (apenas levando em consideração outra linha temporal), para que ocorram comparações de resultados e assim contribuir com mais informações sobre as produções em torno da temática.

\section{REFERÊNCIAS}

ALMEIDA, J. E. F. de et al. Análise dos modelos de avaliação Residual Income Valuation, Abnormal Earnings Growth e Fluxo de Caixa descontado aplicados às ofertas públicas de aquisição de ações no Brasil. Revista de Contabilidade e Organizações, São Paulo, v. 6, n. 16, p. 3-19, dez. 2012.

GєCont, v. 4, n. 1, Floriano-PI, Jan-Jun. 2017. 
ASSAF NETO, A. Contribuição ao estudo da avaliação de empresas no Brasil - uma aplicação prática. Tese de Livre-Docência, Faculdade de Economia, Administração e Contabilidade de Ribeirão Preto da Universidade de São Paulo, São Paulo, 2003.

BRASIL. Comissão de Valores Mobiliários. Instrução n. 361, de 05 de março de 2002 que dispõe sobre o procedimento aplicável às ofertas públicas de aquisição de ações de companhia aberta. Diário Oficial da União, Brasília, 07 mar. 2002. Disponível em:

<http://www.cvm.gov.br/>. Acesso em: 08 jan. 2016.

BRIGHAM, E. F.; EHRHARDT, M. C. Administração Financeira: teoria e prática. São Paulo: Pioneira Thomson Learning, 2006.

COUTO JUNIOR, C. G.; GALDI, F. C. Avaliação de empresas por múltiplos aplicados em empresas agrupadas com análise de cluster. RAM, Rev. Adm. Mackenzie, São Paulo, v. 13, n. 5, p. 135-170, Out. 2012.

CUNHA, M. F. et al. Custo de capital médio ponderado na avaliação de empresas no Brasil: uma investigação da aderência acadêmica e a prática de mercado. Revista Ambiente Contábil, v. 5, n. 2, p. 20-36, set. 2013.

CUNHA, M. F.; MARTINS, E.; NETO, A. A. Avaliação de empresas no Brasil pelo fluxo de caixa descontado: evidências empíricas sob o ponto de vista dos direcionadores de valor nas ofertas públicas de aquisição de ações. Revista de Administração, v. 49, n. 2, p. 251-266, 2014.

DA LUZ, R. M.; GUBIANI, C. A.; PAULO, W. de L.; RAUSCH, R. B. Avaliação de empresas: um estudo bibliométrico sobre as publicações Qualis A de 1998 a 2008 no Brasil. Revista de Contabilidade da UFBA, v. 3, n. 1, p. 34-45, 2009.

DAMODARAN, A. Avaliação de empresas. 2. ed. São Paulo: Pearson Prentice Hall, 2007.

GALDI, F. C.; LOPES, R. F. Avaliação de Ações e Números Contábeis: Aplicação dos Modelos Zhang (2000) e Zhang \& Chen (2007) no Mercado Brasileiro. Revista Brasileira de Finanças, v. 9, n. 1, pp. 131-157, 2011.

GIL, A. C. Como elaborar projetos de pesquisa. 4 ed. São Paulo: Atlas, 2002.

GIRÃO, L. F. A. P.; MARTINS, O. S.; PAULO, E. Avaliação de empresas e probabilidade de negociação com informação privilegiada no mercado brasileiro de capitais. Revista de Administração, v. 49, n. 3, p. 462, 2014.

KOBASHI, N. Y.; SANTOS, R. N. M. Arqueologia do trabalho imaterial: uma aplicação bibliométrica à análise de dissertações e teses. Encontros Bibli: Revista Eletrônica de Biblioteconomia e Ciência da Informação, v. 13, n. esp., p. 106-115, 2008.

LIMA,M. P.; ALMEIDA, V. S. Os analistas sell-side fazem boas previsões de preços-alvo no Brasil? . Revista Brasileira de Finanças, v. 13, n. 3, p. 365-365, 2015.

LIMA, A. C.; CARMONA, C. U. Determinantes da formação do capital intelectual nas 
empresas produtoras de tecnologia da informação e comunicação. RAM, Rev. Adm. Mackenzie, São Paulo, v. 12, n. 1, p. 112-138, Fev. 2011.

MARTELANC, R.; TRIZI, J. S.; PACHECO, A. A. S.; PASIN, R. M. Utilização de metodologias de avaliação de empresas: resultados de uma pesquisa no Brasil. In: Seminário em Administração, 8., 2005, São Paulo. Anais... São Paulo: FEA/USP-SEMEAD, 2005.

MARTELANC, R., PASIN, R., \& PEREIRA, F. Avaliação de empresas: um guia para fusões \& aquisições e private equity. São Paulo: Pearson Prentice Hall, 2010.

MARTINS, E. Avaliação de empresas: da mensuração contábil à econômica. São Paulo: Atlas, 2006.

MELO, D. L. S. ; SILVA, A. P. F. ; FALK, J. A. ; NASCIMENTO, R. G. . Controladoria: um estudo bibliométrico dos artigos publicados em periódicos avaliados pela CAPES. Revista Contabilidade e Controladoria, Curitiba, v. 5, n.3, p. 66-82, set./dez. 2013.

MOREIRA, R. A.; LOCATELLI, R. L.; AFONSO, T. Avaliação e Gestão EconômicoFinanceira de Projetos: Um Estudo Aplicado ao Setor Metalúrgico. Revista de Gestão e Projetos, v. 6, n. 3, p. 28-43, 2015.

OHLSON, J. A. Earnings, Book Values, and Dividends in Equity Valuation. Contemporary Accounting Research, v. 11, n. 2, p. 661-687, 1995

OLIVEIRA, M. R.G.; MEDEIROS NETO, L. B. Simulação de Monte Carlo e Valuation: uma abordagem estocástica. REGE Revista de Gestão, São Paulo, v. 19, n. 3, jul./set. 2012.

PALEPU, K. G.; HEALY, P. M.; BERNARD, V. L. Business Analysis \& Valuation: using financial statements. 2. ed. Ohio: South-Western, 2000

PEREIRO, L. E. Valuation of companies in emerging markets: a practical approach. New York: Wiley, 2002.

RICHARDSON, S.; TUNA, I.; WYSOCKI, P. Accounting anomalies and fundamental analysis: a review of recent research advance. Journal of Accounting and Economics, v. 50, p. $410-454,2010$.

RODRIGUES, P. H. F.et al. Avaliação de empresas start-up por Opções Reais: o caso do setor de biotecnologia. Gest. Prod., São Carlos, v. 20, n. 3, p. 511-523, 2013.

SANTOS, T. B. D.; CUNHA, M. F. Avaliação de empresas: uma análise sob a óptica do "viés de avaliação" nos laudos de oferta pública de aquisição de ações. Revista de Gestão,

Finanças e Contabilidade, v. 5, n. 3, p. 61-74, 2015.

SILVA, E. L.; MENEZES, E. M. Metodologia da pesquisa e elaboração de dissertação. 3. ed. rev. atual. Florianópolis: Laboratório de Ensino a Distância da UFSC, 2001.

SILVA, B. M.; SANTOS, N. J. D. Apuração de haveres de sócio: o caso de uma empresa do comércio varejista da grande Florianópolis. Revista Catarinense da Ciência Contábil, v. 12, n. 34, p. 53-65, 2013. 
TOZONI-REIS, M. F. C. Metodologia da pesquisa. Curitiba: IESDE Brasil SA, 2009. 